\title{
Frequency dependence of gel time in thermosetting resins ${ }^{\dagger}$
}

\author{
ANAND PAL SINGH* and H R ANAND \\ School of Basic and Applied Science, Thapar Institute of Engineering and Technology, \\ Patiala 147001 , India \\ *Present address: Post Graduate Department of Chemistry, Khalsa College, Amritsar \\ 143001 , India
}

\begin{abstract}
tbstract. The increasing use of thermosetting resins in fibre reinforced composites for structural and insulating applications has necessitated the need to understand the process of crosslinking and systematics of curing time of resins with and without the reinforcing fine fibre tilaments. We have employed dynamical measurements of loss tangent and dielectric constant uver the frequency range $1 \mathrm{kHz}-100 \mathrm{kHz}$ for investigating cure characteristics of composites. After adding crosslinking agents to resinous matrices (epoxy and polyester styrenated alkyd resins) the time variation of dielectric parameters was recorded until the completion of curing. Ihe resin flow, the onset of gelation and completion of curing were unambiguously mapped by variations in dielectric parameters. Onset of gelation coincided with precipitous fall of dielectric constant and resonant maximum of dielectric loss. Asymptotic stabilization of dielectric parameters signalled completion of curing. The gel time and curing time were found w) be substantially larger for fibre reinforced resins as compared to pure resins. The variation of electrically determined gel and cure time with frequency of ac signal employed and on the thre fraction in the composite are discussed in the paper. The present study shows that if dielectric measurements are to be employed to infer the completion of curing, low frequency measurements would be needed to correctly infer onset of gelation and time for curing.
\end{abstract}

Keywords. Thermosets; composite materials; dynamic dielectric analyses; gel time; curing time.

\section{Introduction}

Dynamic dielectrometric analysis is a well known instrumental method of materials evaluation (Kranbuehl 1980; Bidstrup et al 1986; Kranbuehl et al 1986; Sanjana 1986; Songzhen et al 1988; Gotro and Yandrasite 1989). However, there is scant literature relating frequency of ac signal to cure parameters of polymers. In the present study dielectrometrically determined gel time, a key parameter of the process of crosslinking, has been examined in relation to frequency of the test signal. This paper reports on frequency dependence of dielectrically determined gel time which is the time corresponding to the maximum rate of decrease in permittivity or time corresponding to the dipolar relaxation peak. An attempt has been made to correlate the results of ac measurements with dc conductivity. Gel time is found to correlate negatively with frequency of ac signal and its limiting value for lower frequencies corresponds to gel time inferred from static (dc) measurements.

\section{Experimental}

Dynamic dielectric measurements were made using a multi-frequency LCR meter (Hewlett-Packard 4274-A) capable of measuring real and imaginary components of

'Paper presented at the poster session of MRSI AGM VI, Kharagpur, 1995

* To whom all correspondence should be addressed. 
permittivity over the range $10 \mathrm{~Hz}-100 \mathrm{kHz}$ at 11 spot frequencies. The measurements on pure resin and resin based fibrous composites (Zee et al 1989) were made by sandwiching the resin and resin/fibre mix between two copper sheet electrodes inside a sample holder. Sample holder geometry has been described in our previous publication (Anand and Singh 1993).

Two resin systems were investigated--epoxy and polyester. The polyester styrenated alkyd was cured with varying proportions $(2-4 \%)$ of methyl ethyl ketone peroxide as catalyst (initiator) and cobalt naphthenate salt as accelerator (activator) while the epoxy resin (Ciba Geigy LY 557) was cured with $10 \%$ by weight of the liquid aliphatic amine hardener (HY 951). The resin and additives were weighed to their desired molar ratio on an electrical single pan (Sartorius) balance. Soon after mixing the crosslinking additives with resin. the syrupy liquid was poured into the test cell. For fibre glass filled specimens, layers of chopped strand mat (CSM) of glass fibre were wetted in resin/hardener mix and pressed together to achieve a $65 \%$ resin-35\% glass volume proportion.

All electrical measurements were made using an electrically shielded system to prevent the interference of stray currents and pickups. A four terminal pair-type fixture equipped with four BNC (m) terminated-coaxial test leads was used. These leads were used to attach the shielded system to the HP 4274 LCR meter. This arrangement was made to avoid stray capacitances by grounding the metallic chamber. The magnitude of capacitance and loss factor were measured at various frequencies $(2 \mathrm{kHz}-100 \mathrm{kHz})$ in the parallel equivalent circuit mode.

\section{Results and discussion}

Figures 1-6 show variation of dielectric loss factor and dielectric constant against

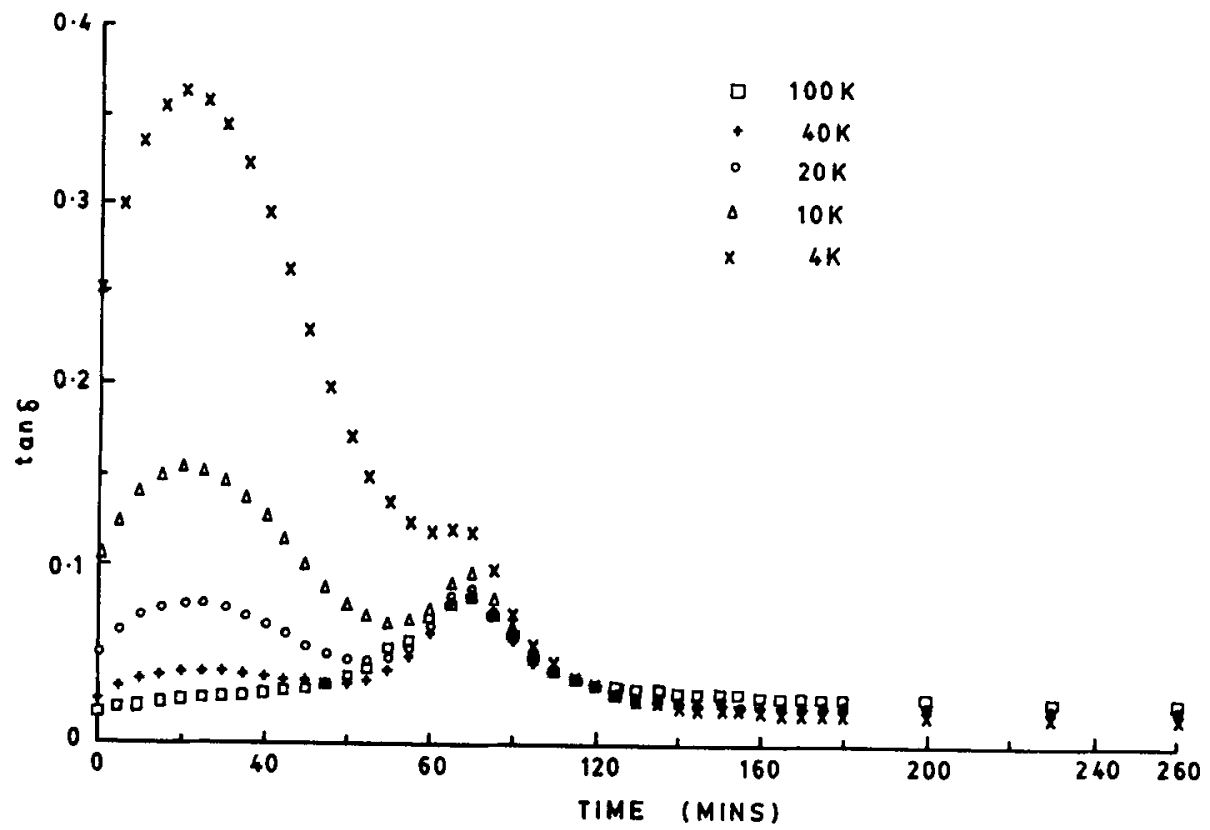

Figure 1. Loss factor vs curing time for epoxy resin. 


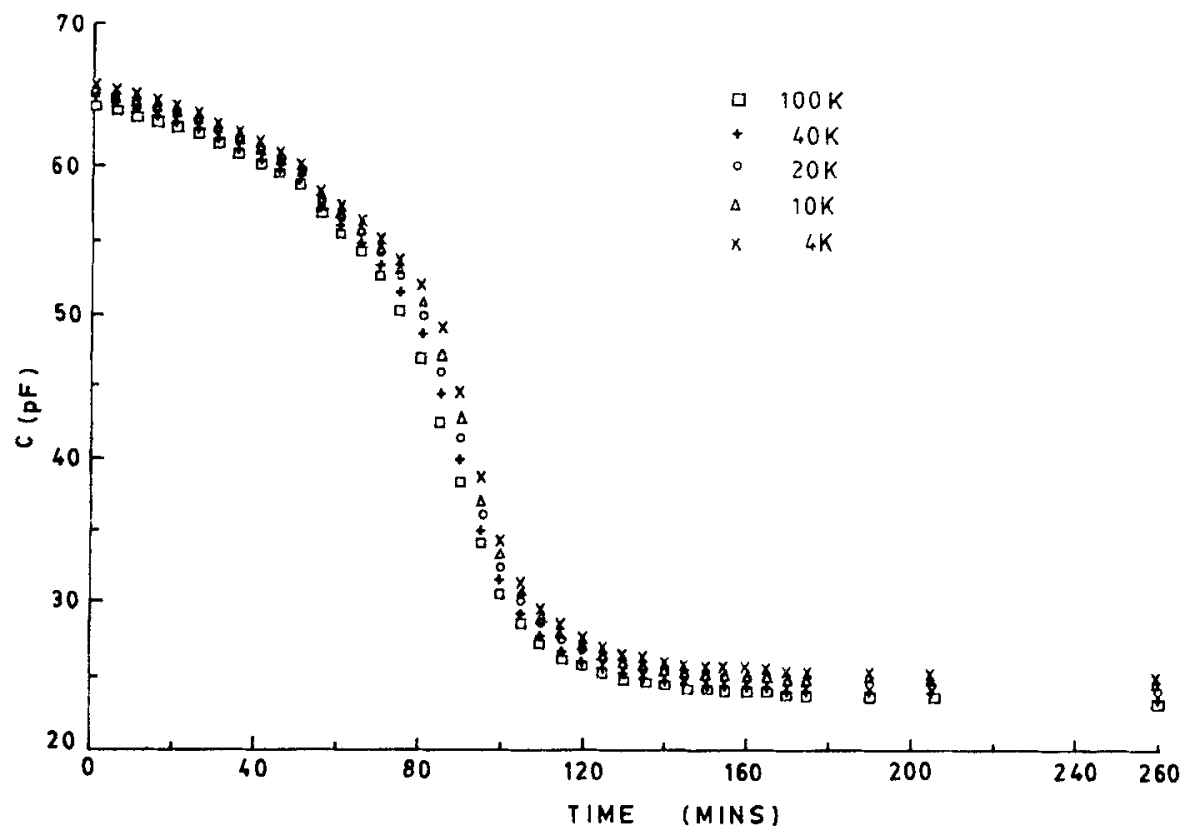

Figure 2. Lapacitance vs curng time for epoxy resin.

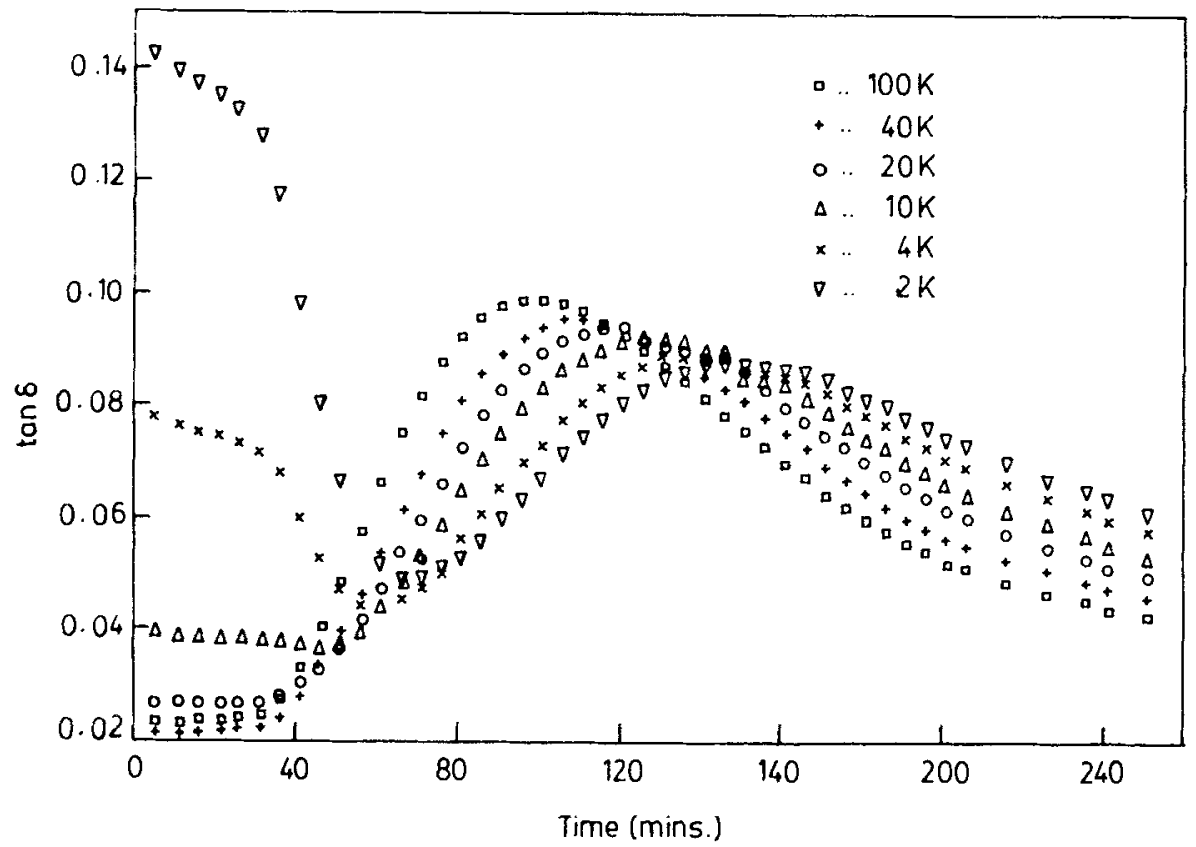

Figure 3. Luss factor vs curng tme for polyester resin ( $2 \%$ catalyst: $2 \%$ accelerator).

curing time for epoxy and polyester resin at different test frequencies. All dielectric loss factor curves reach a maximum first, whose peak height is frequency dependent, followed by a well-defined minimum and a second peak before they asymptotically 


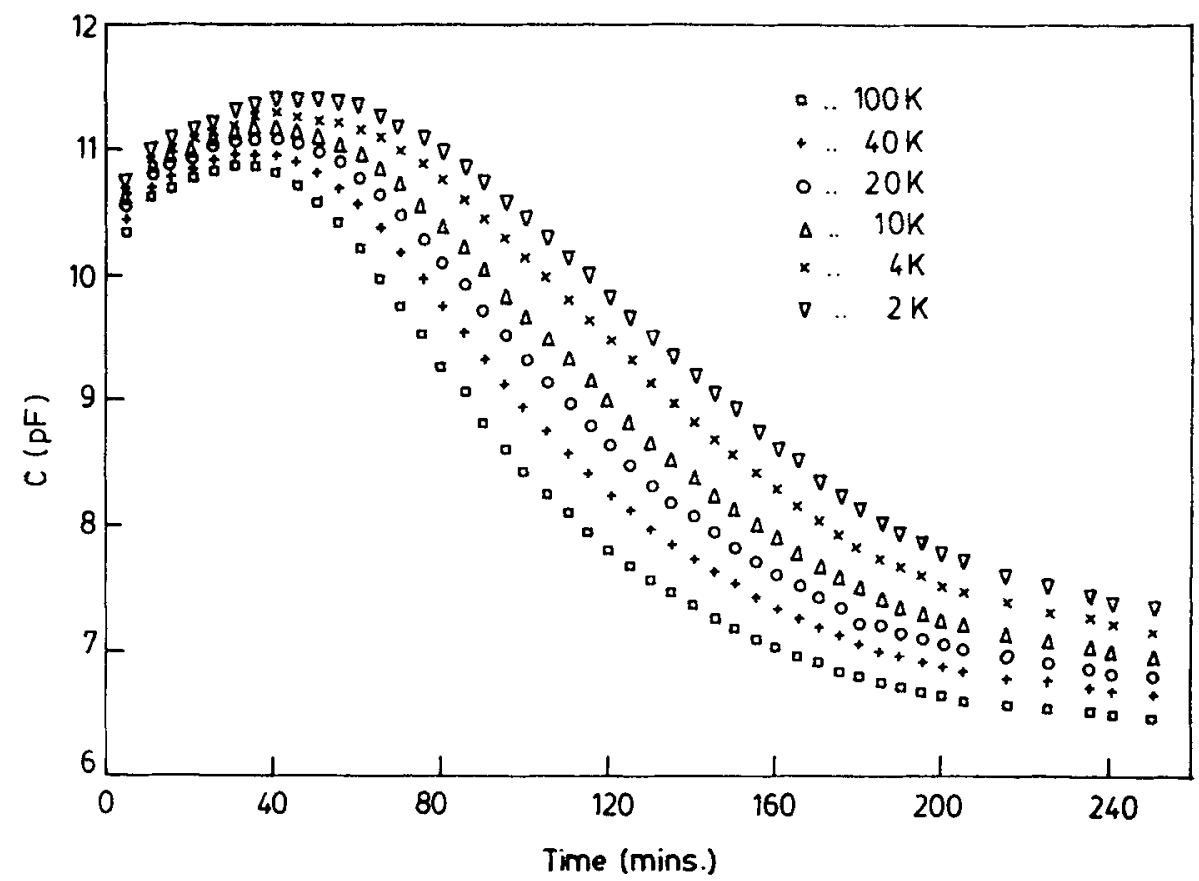

Figure 4. Capacitance vs curing time for polyester resin ( $2 \%$ catalyst: $2 \%$ accelerator).

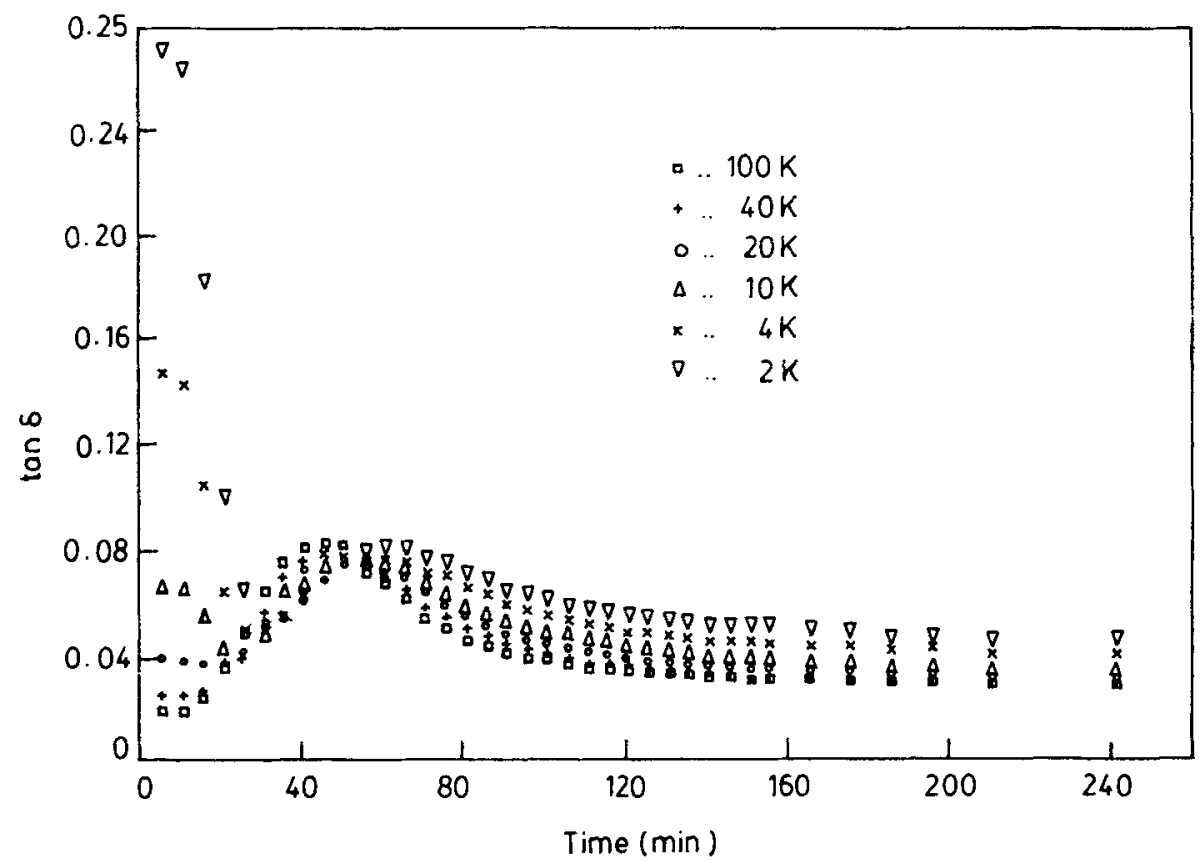

Figure 5. Loss factor vs curing time for polyester resin ( $4 \%$ catalyst: $4 \%$ accelerator). 


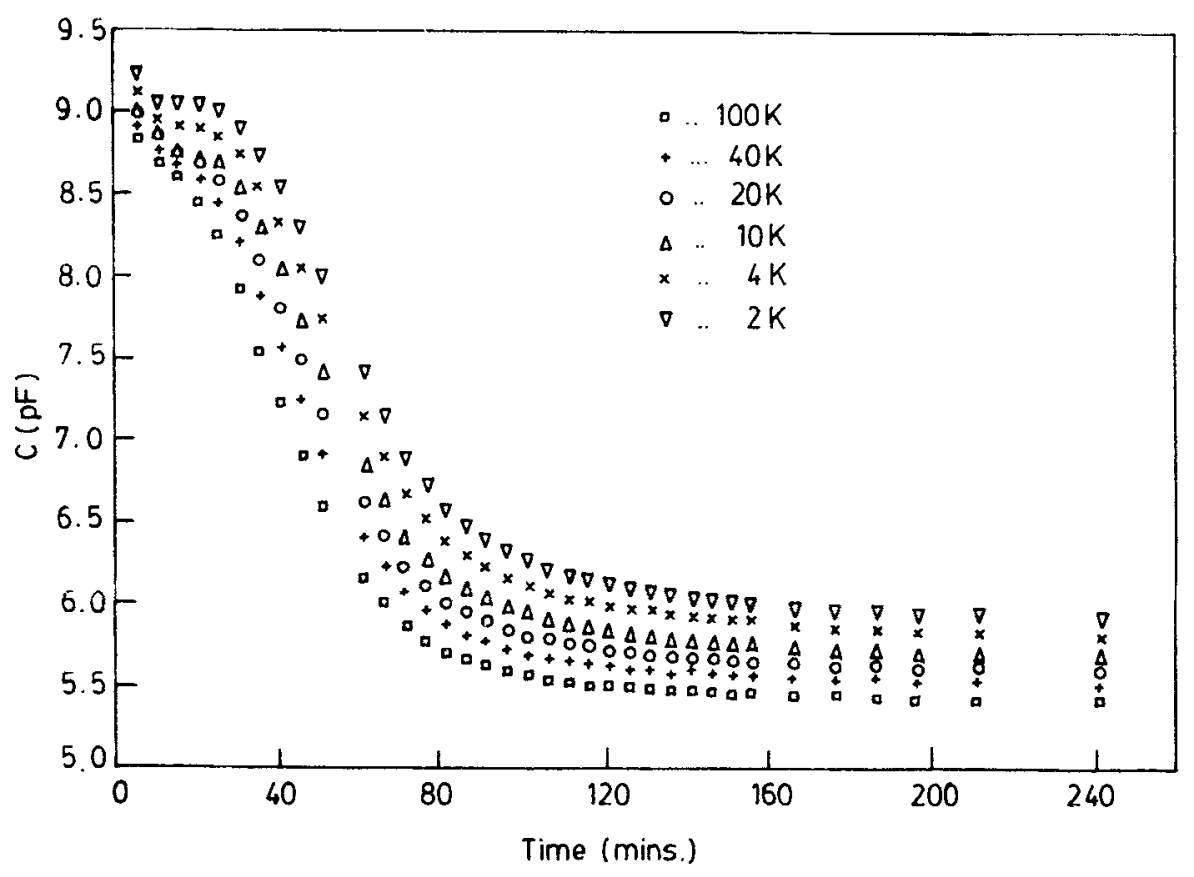

Figure 0 . Capacitance vs curıng tıme for polyester resin (4\% catalyst: $4 \%$ accelerator).

approach a final steady value. The loss maxima corresponding to higher frequencies is seen to be reached somewhat earlier. The dielectric loss factor is composed of two components, one due to dipole relaxation and the other arising from bulk (or ionic) conduction. The ionic conductivity effect is more pronounced at lower frequencies. During the exothermic rise, the temperature is the highest and viscosity is least. The ions are most mobile and the contribution from ionic conduction becomes significant. The ionic conduction mechanism is mostly attributed to residual ionic species formed during monomer synthesis (Senturia and Sheppard 1986). At elevated temperatures the loss factor (ionic conductivity) trucks with the mobility of the ionic impurities in the network. At higher temperature the difference in loss factor between high and low applied frequency conditions is large as evident in the first (exothermic) peak. The loss difference between high and low frequencies is more subdued when the sample has almost stabilized in temperature i.e. 2nd peak. The occurrence of this peak may be attributed to the motion of polar groups in response to the applied electric field, but their motions lag behind the field causing a continuous loss of energy. The loss of energy goes through a maximum and then decreases as molecular motion becomes more and more restricted making alignment with applied field more difficult. At the onset of gelation (i.e. the beginning of the 2 nd peak in loss factor data) there is a rapid drop in dielectric constant. As the maximum rate of decline in dielectric constant coincides with the second peak for loss factor, this can be safely attributed to dipolar relaxation. As the curing is completed the systems become rigid and dipoles become immobile resulting in a stabilized (low) value for dielectric constant and loss factor.

Figures $7-12$ show dielectric loss and dielectric constant variations vs curing time for glass fibre ( $35 \%$ by volume) reinforced epoxy and polyester resin. 


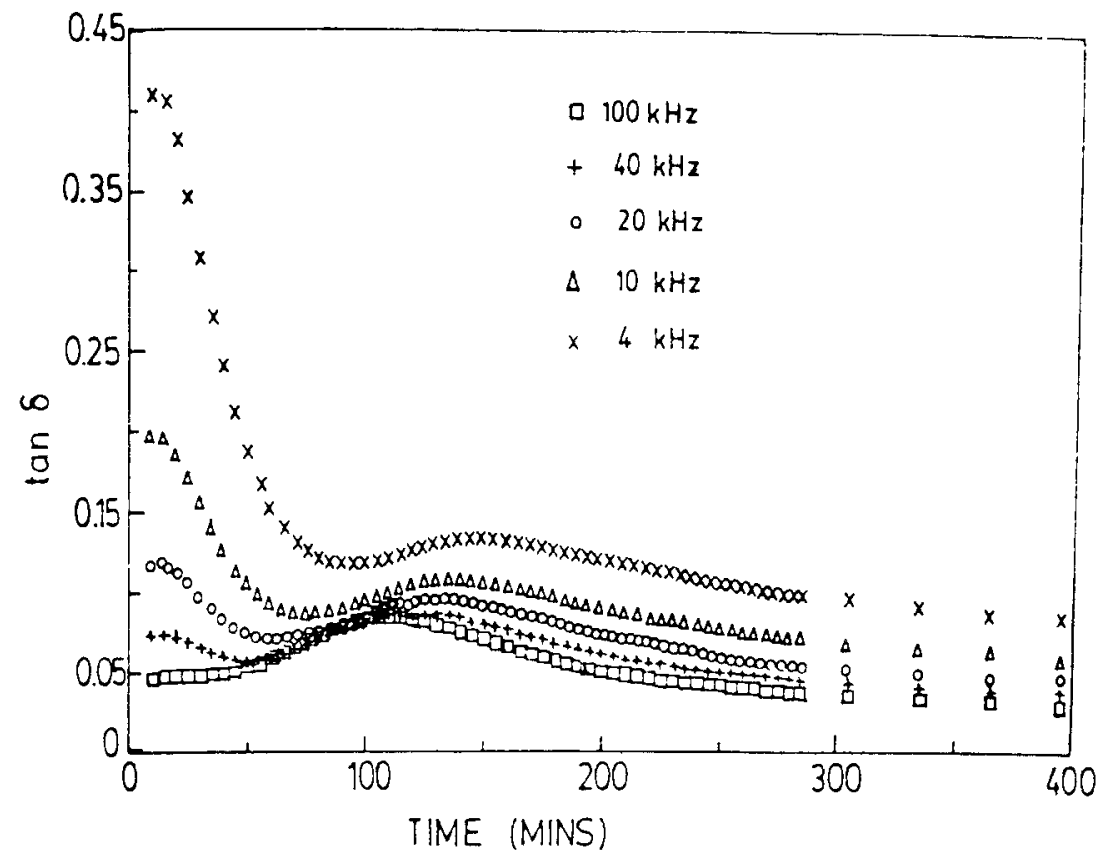

Figure 7. Loss factor vs curing time for glass fibre reinforced epoxy resin.

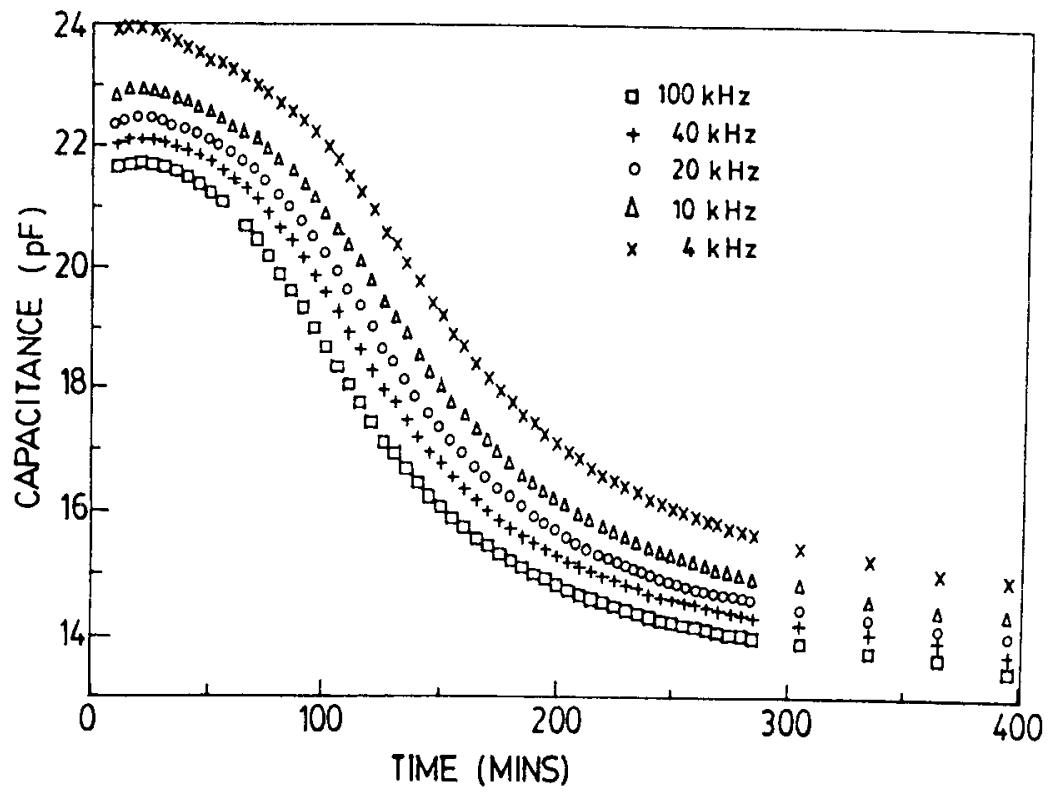

Figure 8. Capacitance vs curing tıme for glass fibre reinforced epoxy resin.

The general trend of loss factor and dielectric constant data for (near isothermal) glass mat reinforced specimens is similar to the unfilled resin system (figures 1-6) except that curing takes substantially longer time in the former. The curing time for glass 


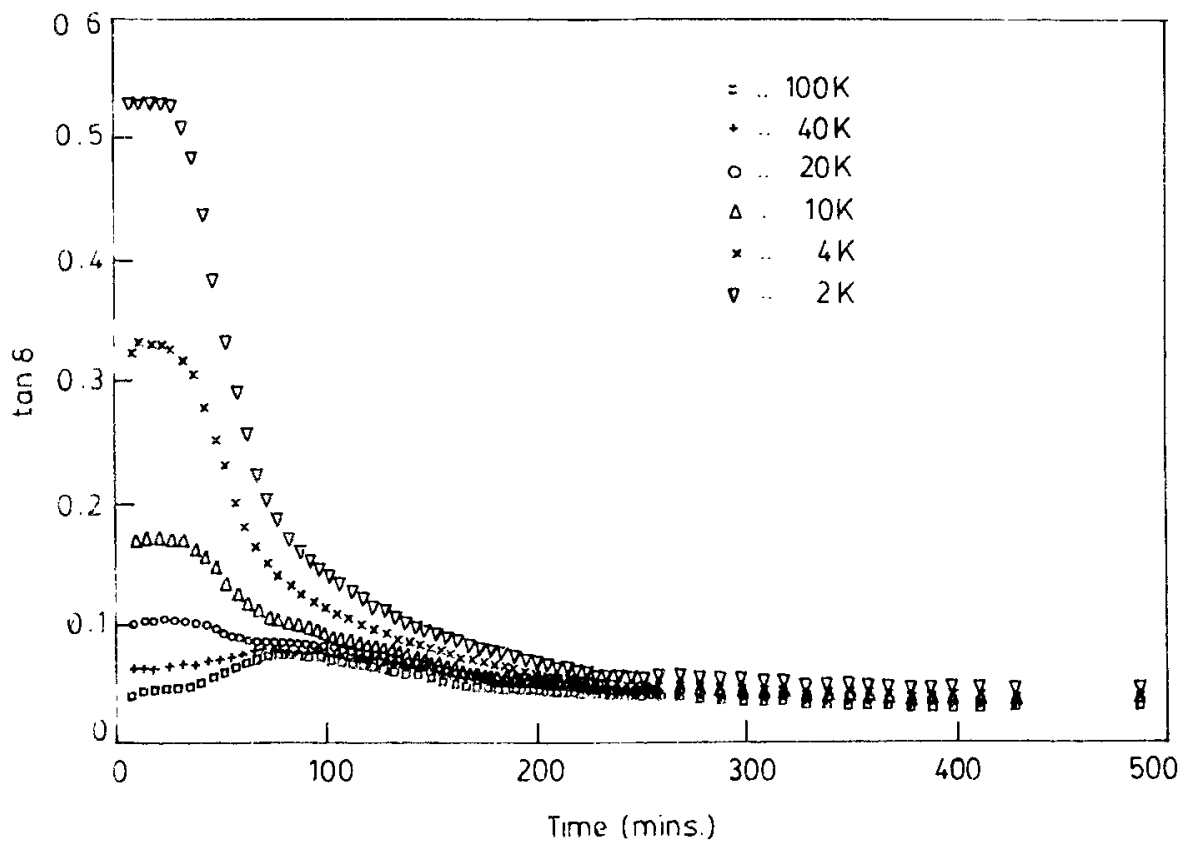

figure 9. L_uss lactor vs cuungr ume for glass tibre remforced polyester resin ( $2 \%$ catalyst: $2 \%$ liceleratort.

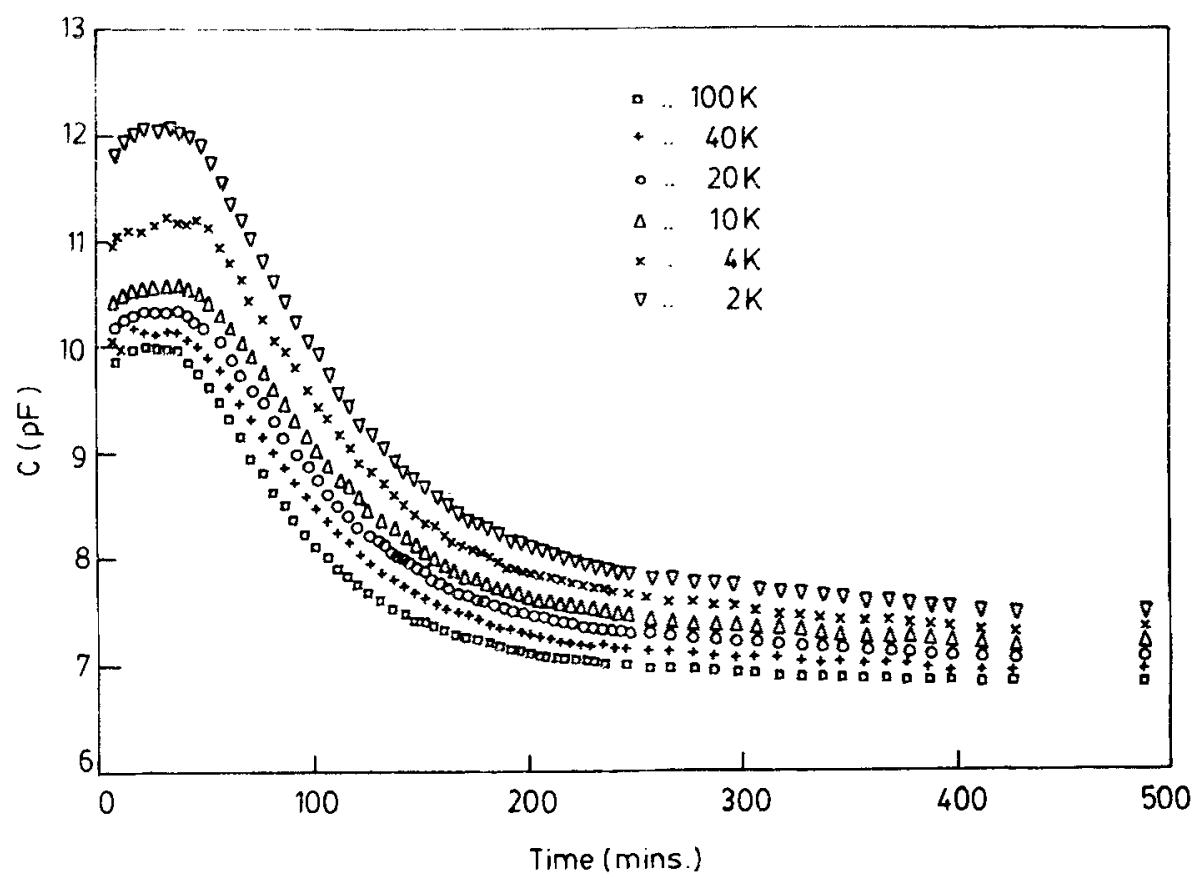

Figure 10. Capacitance vs curng time for glass fibre reinforced polyester resin (2\% catalyst: $2 \%$ accelerator). 


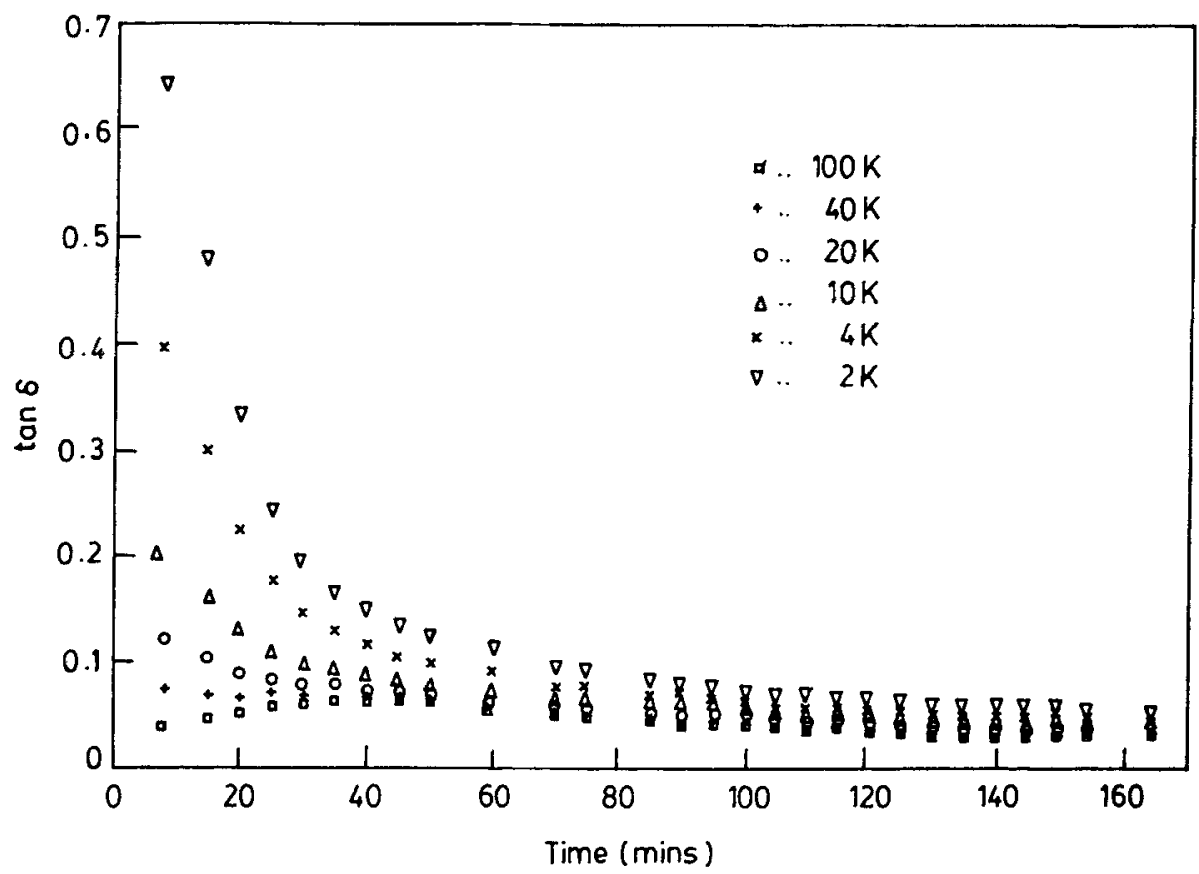

Figure 11. Loss factor vs curing time for glass fibre reinforced polyester resin (4\% catalyst: $4 \%$ accelerator).

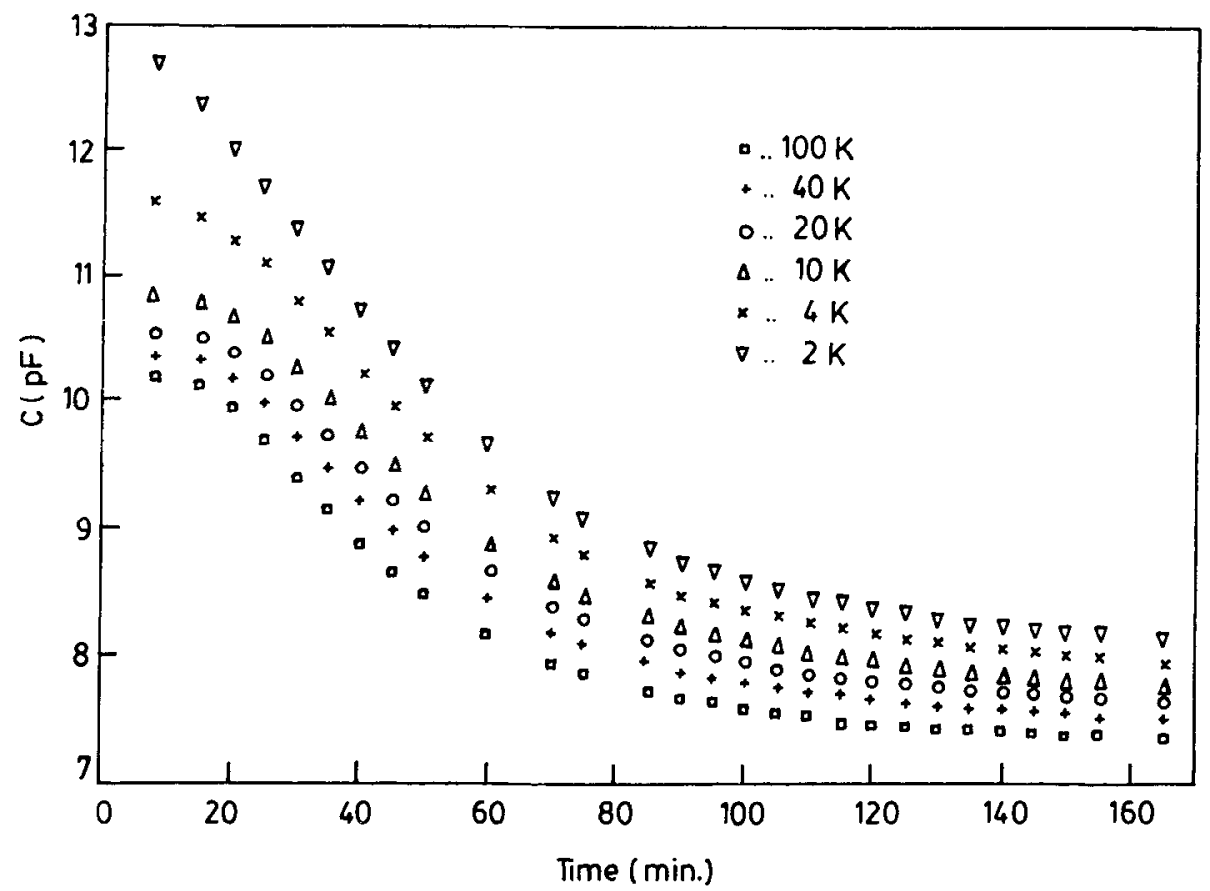

Figure 12. Capacitance vs curing time for glass fibre reinforced polyester resin (4\% catalyst: $4 \%$ accelerator). 
reinforced epuxy specimens was nearly twice the time for corresponding unfilled resin. In comparison, polyester resin based composites showed nearly same curing time as for corresponding unfilled resins.

During curing of polyester resin for varying proportions of methyl ethyl ketone peroxide and cobalt naphthenate salts the dielectric loss peaks (indicating gelation) corresponding to high frequencies registered their maxima earlier than that of low frequency peaks. As explained earlier the first maxima in the dielectric loss factor is due to the ionic component, which dominates at low frequencies, low viscosities and high temperatures (Sanford and McCullough 1987), while the second maxima is due to the dipolar component arising from rotational movement of molecular dipole moments. This dipolar term dominates the dielectric loss signal at higher frequencies.

Curing time is seen to be strongly dependent on proportions of catalyst and accelerator. Proportion of catalyst, however, seems to have greater bearing on curing time than the proportion of accelerator.

There is a small point of difference between the results of dielectric constant (figures 2,4 and 6) for unfilled epoxy and polyester resin during the exothermic rise. The permittivity of the unfilled polyester resin initially increases before starting to drop during the course of the reaction resulting in a gentle hump during the exothermic rise. This is more prominent at lower frequencies (figures 4 and 6 ) and for lower proportions of catalyst and accelerator. In epoxy resin (figure 2) where only one proportion of hardener $(10 \%)$ was employed no such hump is evidenced. The higher magnitudes of dielectric constant at lower frequency reflect the role of space charge polarization which have typically low resonance frequency.

The changes in electrical properties of epoxy and polyester system point to a threestep process of curing. During first phase (resin flow) the dielectric loss exhibits a maximum. This is followed by gelation which results in a dipolar resonance peak for dielectric loss, and rapid decline in relative permittivity. The third phase of eventual completion of curing is signalled by an asymptotic stabilization of all electrical parameters. The dielectrometrically determined gel time would be the time corresponding to dipolar resonance peak.

In terms of curing process, crosslinking starts independently at a number of places in the resin/hardener liquid (analogous to the growth of grains in a melt in the process of freezing). Small regions (floccules) of crosslinked resin in a liquid matrix of resin/hardener may be viewed as a two-phase system resembling a gel. With time crosslinking spreads consuming more and more of the liquid phase and in the end a rigid (cured) specimen is realized.

A look at figure 3 shows that lower frequencies reach their second loss (dipolar) maximum at substantially later times. Lower frequency peak corresponds to resonance with respect to larger floccules (crosslinked phase) involving longer chains of larger molecular weight which will take longer time to form. In epoxy system, frequency dependence ('dispersion') is rather small in absence of fillers (figure 1), while in polyester system, frequency dependence is rather large in absence of glass fibres.

The gel time for different ac frequencies has been least squares fitted according to the equation:

$$
t(f)=t(1)+m \log f
$$

where $t(f)$ is ac gel time and $t(1)$ corresponds to gel time for $1 \mathrm{~Hz}$ (approximating to dc gel time). From the intercept $(t(1))$ of the linear plot, one can predict the dc gel 


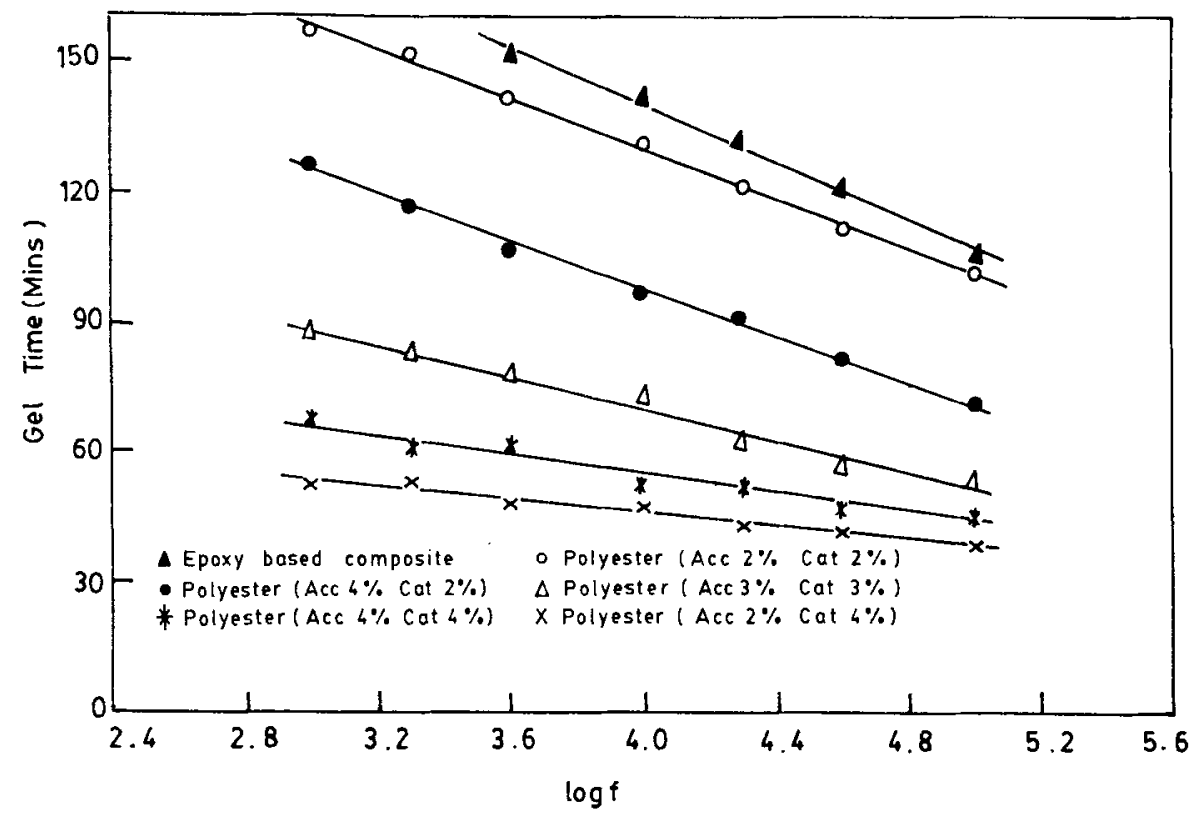

Figure 13. Plot of frequency vs gel time.

Table 1. Slope and intercept of $\log$ of frequency curve vs gel time.

\begin{tabular}{lrrr}
\hline Sample & Slope & \multicolumn{1}{c}{$t(1)$} & \multicolumn{1}{c}{$t(50)$} \\
\hline Epoxy based composite & -32.314 & 268.006 & 213.109 \\
Polyester (Acc2\% Cat2\%) & -28.484 & 243.434 & 195.007 \\
Polyester (Acc4\% Cat2\%) & -26.846 & 204.784 & 159.173 \\
Polyester (Acc3\% Cat3\%) & -18.237 & 142.298 & 111.315 \\
Polyester (Acc4\% Cat4\%) & -10.756 & 97.299 & 79.024 \\
Polyester (Acc2\% Cat4\%) & -7.525 & 75.463 & 62.678 \\
\hline
\end{tabular}

time for the given resin system. Slope $m$ of this plot depends on the nature of the resin/hardener system. Magnitude of (negative) value of slope indicates the degree of dispersion i.e. the extent to which gel time reduces at higher frequencies. In case of epoxy based composite only one resin/hardener composition was employed in the present study while for polyester system five different proportions of catalyst and accelerator were used. Therefore the latter system results in five gel time vs log of frequency plots (figure 13).

Table 1 gives extrapolated gel time for $1 \mathrm{~Hz}$ and $50 \mathrm{~Hz}$. The former is expected to be close to dc gel time observed in dc measurements. There is a good agreement between expected and observed gel time for dc for epoxy system and most of the polyester preparations. The maximum departure is in the case of polyester preparation incorporating $4 \%$ catalyst and $2 \%$ accelerator. The scatter in gel time data for higher catalyst preparations results in a much larger uncertainty in the extrapolated intercept i.e. $t(1)$. An examination of extrapolated gel time for $50 \mathrm{~Hz}, t(50)$, shows a relatively better 


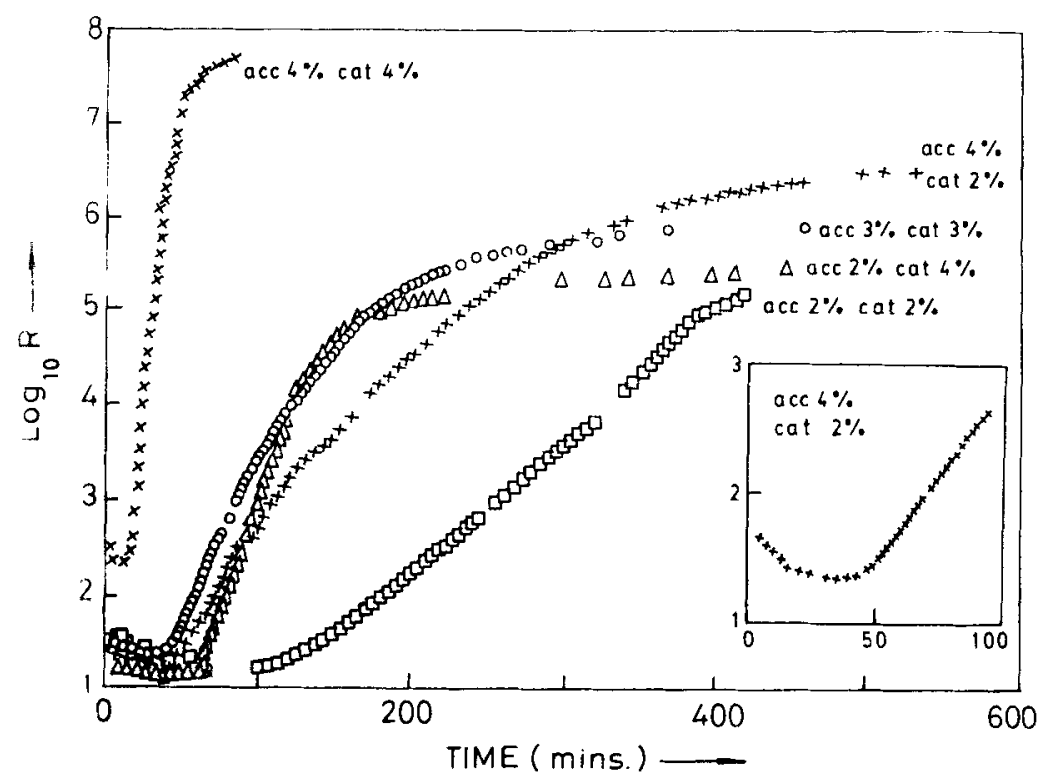

Figure 14. Resistance vs curıng time for polyester resin.

agreement. Poor agreement in extrapolated gel time values from a log of frequency plot is not entirely unexpected in the low frequency $(1 \mathrm{~Hz})$ range far away from the experimental frequency range $(1 \mathrm{kHz}-100 \mathrm{kHz})$. Thus, overall there is a credible correlation between dc (measured) gel time and low frequency gel time inferred from higher frequency ac gel times.

The resistance measurement is also able to identify time domains of resin flow, gelation and curing time (figure 14). The gel time can be determined from the resistance vs time profile as the time corresponding to steepest rise (maximum slope). Gel time corresponding to low frequency limit and curing time for complete curing can be explicitly determined from dc resistance vs time study. These parameters can be inferred from ac studies by extrapolation to low frequency limit. On this account dc measurement would seem to be simpler and thus have an edge over ac measurement. However if additional information regarding dipolar relaxation times is to be studied, only ac measurements are the answer. Thus the dc and ac have their own distinctive redeeming features.

\section{Conclusions}

Dynamical dielectric measurements unambiguously map the onset of gelation and curing of resins. Gel time and curing time can be inferred for resins and resin based composites from dielectric measurements. The present work shows that time of onset of gelation as inferred from ac measurements is, however, a frequency dependent parameter. This study suggests that dynamical dc electrical monitoring should be employed to ensure complete curing of a particular polymer system since gel time of dc measurements is substantially larger than the corresponding gel time as determined from ac measurements. 


\section{References}

Anand H R and Singh Anand Pal 1993 J. Polym. Mater. 10161

Bidstrup W W, Sheppard N F and Senturia S D 1986 Polym. Engg. \& Sci. 26358

Gotro J and Yandrasite M 1989 Polym. Engg. \& Sci. 29278

Kranbuehl D E 1980 Developments in reinforced plastics-5 (ed.) G Pritchard (London: Elsevier) p 181

Kranbuehl D E, Delos S E. Hoff M S, Whitham M E and Weller L M 1986 Proc. of the second conference on advanced composites (America: ASM International Publishers) p 61

Sanford W M and McCullough R L 1987 Proc. of the American society for composites second technical conference (USA) p 21

Sanjana Z N 1986 Polym. Engg. \& Sci. 26373

Senturia S D and Sheppard N F 1986 Adv. Polym. Sci. 801

Songzhen Wu, Gedeon S and Fouracre R A 1988 IEEE Trans. Elect. Insul. 23409

Zee R H, Huang Y H, Chen J J and Jang B Z 1989 Polym. Comp. 10205 